\title{
CHARACTERISTIC STUDY OF MHD MINIPUMP
}

Je -Ee Ho

Department of Mechanical Engineering, National Ilan University, Ilan, Taiwan, R.O.C., jeho@niu.edu.tw

Follow this and additional works at: https://jmstt.ntou.edu.tw/journal

Part of the Mechanical Engineering Commons

\section{Recommended Citation}

Ho, Je -Ee (2008) "CHARACTERISTIC STUDY OF MHD MINIPUMP," Journal of Marine Science and Technology. Vol. 16: Iss. 3 , Article 9.

DOI: $10.51400 / 2709-6998.2015$

Available at: https://jmstt.ntou.edu.tw/journal/vol16/iss3/9

This Research Article is brought to you for free and open access by Journal of Marine Science and Technology. It has been accepted for inclusion in Journal of Marine Science and Technology by an authorized editor of Journal of Marine Science and Technology. 


\title{
CHARACTERISTIC STUDY OF MHD MINIPUMP
}

\author{
$\mathrm{Je}-\mathrm{Ee} \mathrm{Ho}^{*}$
}

Key words: MHD minipump.

\begin{abstract}
The present study aims to develop and discuss the availability of the Magneto Hydrodynamics Minipump (MHD) as a miniaturizative part specified for precise manufacturing or medical application. Based on the MHD theory, an available working current $(1.8 \mathrm{~A})$ induced by lower voltage $(0-12 \mathrm{~V})$ and weaker magnetic intensity $(0.04 \mathrm{~T})$ meets the need in mini-system operation. However, precise estimations of working capacity, maximum head, dynamic head, and static head in various electrolyte concentrations are necessary. Furthermore, this work also provides a simplified analytic model with a static balance between Lorentz force, surface tension force, and body force. Compared with analytic solutions and experimental results, the static head depends on the operating voltage, and the higher dynamics head is produced instantly in the working medium of $\mathrm{NaCl} \cdot \mathrm{H}_{2} \mathrm{O}$ which is more appropriate for the impulse pump.
\end{abstract}

\section{INTRODUCTION}

Magneto hydrodynamics (MHD), which primarily describes the flow dynamics by coupling the interaction of electrically conducting current and electro-magnetic intensity, has gradually drawn public attention to the field of micro fluids. Though the basic MHD principle began to be discussed in the last decade, the effective application in various fields has been studied only recently. Geng, X., Yuan, H. and Oguz, H.N. [1] calculated the size of bubbles in the pumping process and discussed the availability of the MHD pump in the micro channel. Huang et al. [2] also demonstrated the pumping characteristic with several aqueous solutions and found that the formation of bubbles at the electrode significantly affected the performance of the pump. Jang and Lee [3] published a paper on the MHD minipump and evaluated its performance by measuring the pressure head and regulating the voltage range from $10-60 \mathrm{~V}$ under a magnetic flux density of 0.19-0.44T. Luis et al. [4] proposed a finite element method to simulate the MHD duct flow with a formulation of a

Paper submitted 05/11/07; accepted 09/28/07. Author for correspondence: Je-Ee Ho (e-mail:jeho@niu.edu.tw).

*Department of Mechanical Engineering, National Ilan University, Ilan, Taiwan, R.O.C. penalty function. Extended analysis proved the Hartman number to be a key parameter which significantly influenced the flowing behavior of the electrical conducting liquid. The simulation results showed that the restricted Hartman number $10^{2} \sim 10^{3}$ would achieve a stable solution which gave a reasonable flow distribution. Wang et al. [5] provided a simplified 2-D MHD model assuming a steady state with an incompressible and fully developed flow. Under this condition, the Lorentz force was converted into pressure terms and occurring frictional effects on the channel sidewalls were also taken into account.

This research sets up an experimental model with two electrolytes in different concentrations. The designed minipump with a distance of 3 millimeter between the electrodes operates in smaller input voltage (may be in series with the usage of $3 \mathrm{~V}$ batteries); which is quite different from the operation in higher voltages or stronger magnetic fields in previous experiments. Moreover, an analytic solution with static head is also derived to identify the experiment results in this study.

\section{FUNDAMENTAL ANALYSIS}

The experimental apparatus is sketched in Figs. 1 3, which consists of four major systems: power supply system, hydraulic head measuring tube, image recording system and test channel. A power supply (Gpc-3030) with a voltage range of $0 \mathrm{~V} \sim 30 \mathrm{~V}$, and a current between $0 \mathrm{~A} \sim 3 \mathrm{~A}$ was used. Copper sheets with a physical dimension of $60 \times 3 \times 0.2 \mathrm{~mm}^{3}$ placed on the top and bottom of a test channel made up by thin acrylic plates were used as electrodes, and then the channel sidewalls were equipped with high-density magnetism with a magnetic flux of $0.04 \mathrm{~T}$ (Fig. 2). To measure the static head of electrolyte, an acrylic measuring tube with a diameter of $1 \mathrm{~mm}$ and an inclined angle of 30 degree was sealed at the right side (Fig. 3). A camcorder (DCR-HC 20) connected to a personal computer through a traducer wire captured the recorded image and transmitted it to the personal computer in real time.

Initially, the test channel was filled with electrolyte and surface tension force elevated the liquid to height $h_{0}$. This is defined as the equilibrium between surface tension and elevated liquid weight.

$$
\sigma 2 \pi r \sin \theta=\rho \pi r^{2} g h_{0}
$$




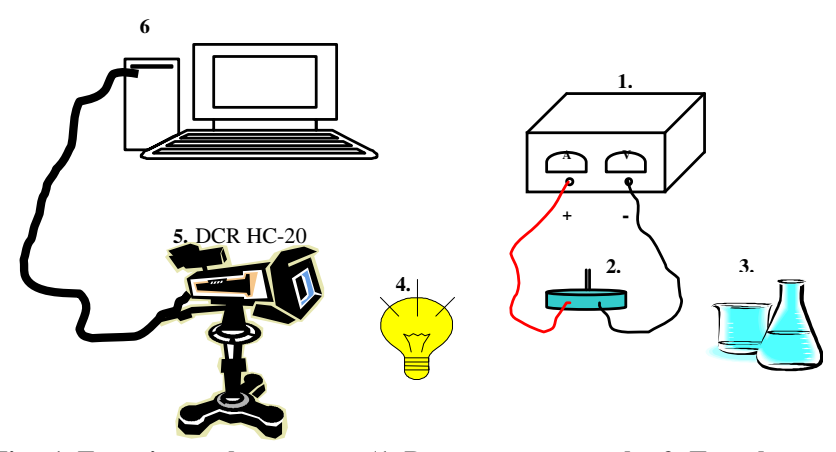

Fig . 1. Experimental apparatus (1. Power source supply; 2. Test channel; 3. $\mathrm{CuSO}_{4} \cdot \mathbf{5} \mathrm{H}_{2} \mathrm{O}, \mathrm{NaCl}$; 4. Halogen lamp; 5. Camera DCR HC-20; 6. Personal computer).

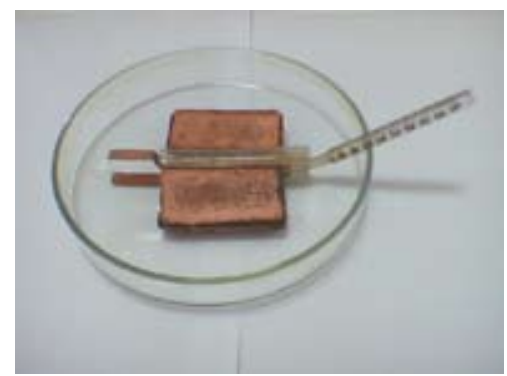

Fig. 2. Photograph of test channel.

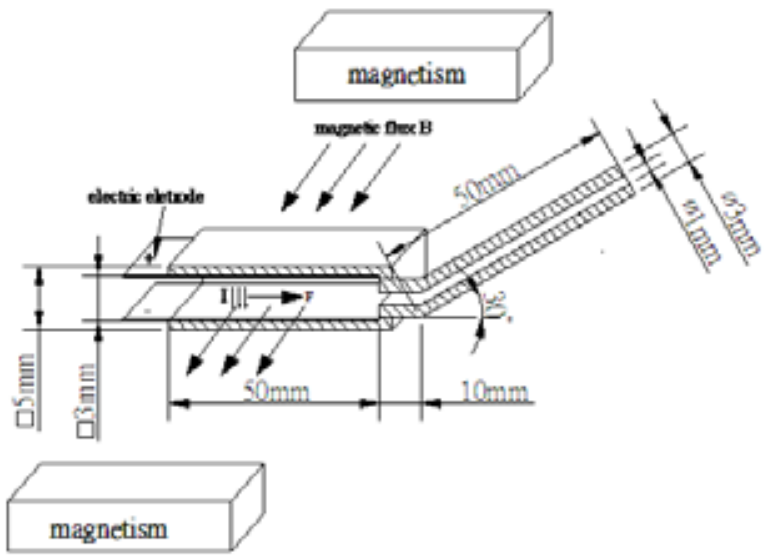

Fig. 3. Cross section view of test channel.

When the external voltage induced the parallel working current, the electrolyzing process started simultaneously. Once the induced current interacted with the normal magnetic field, the pumping motion began. This motion immediately raised the working medium until the final balance between body force, surface tension force, and Lorentz force was reached.

$$
J \times B \times L+\sigma 2 \pi r \sin \theta=\rho \pi r^{2}\left(h_{0}+\Delta h\right) g
$$

Substituting (1) into above equation, the final expression could be simplified as:

$$
\Rightarrow J \times B \times L=\rho \pi r^{2} \Delta h g
$$

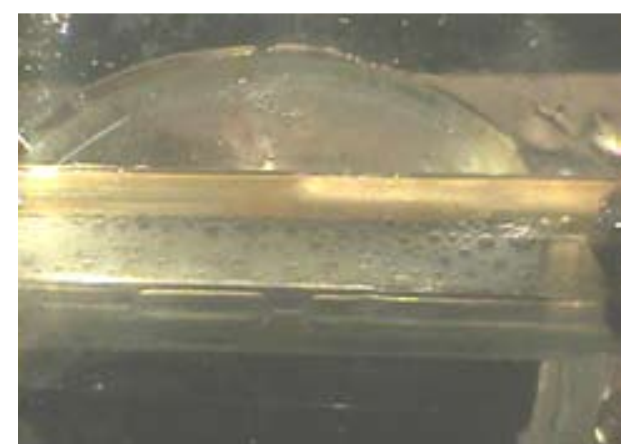

Fig. 4. Bubble's generation for $\mathrm{NaCl} \cdot \mathrm{H}_{2} \mathrm{O}$ at $\mathrm{t}=5 \mathrm{~s}$ under an applied voltage of $9 \mathrm{~V}$.

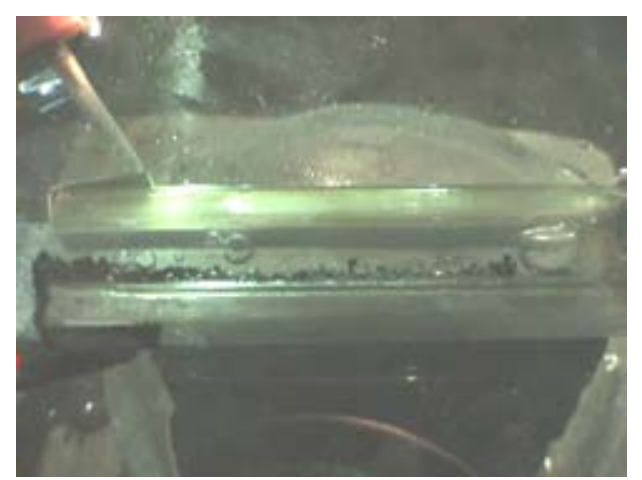

Fig. 5. Bubble's generation for $\mathrm{CuSO}_{4} \cdot 5 \mathrm{H}_{2} \mathrm{O}$ at $\mathrm{t}=5 \mathrm{~s}$ under an applied voltage of $9 \mathrm{~V}$.

\section{RESULT AND DISCUSSION}

\section{Induced Current and Maximum Head with Time}

The significant difference between the two aqueous solutions, $\mathrm{NaCl}$ aqueous solution and $\mathrm{CuSO}_{4} \cdot 5 \mathrm{H}_{2} \mathrm{O}$ aqueous solution, used in this study is the amount of bubbles.

Figure 4 shows that $\mathrm{NaCl}$ solution easily produces massive bubbles (Fig. 4), and then $\mathrm{CuSO}_{4} \cdot 5 \mathrm{H}_{2} \mathrm{O}$ produces less under a voltage of $9 \mathrm{~V}$, and magnetic flux of $0.04 \mathrm{~T}$ (Fig. 5). Due to the electrolyte with higher electric conductivity $(100 \mathrm{~ms} / \mathrm{cm})$ in $5 \%$ $\mathrm{NaCl}$ solution, the induced current jumped up to $1.8 \mathrm{~A}$ immediately within the first second (see Fig. 6) resulting in the formation of a massive amount of tiny bubbles in the channel, which reduces the electrical conductivity in the working medium, and made the induced current drop from 1.8 A to $0.25 \mathrm{~A}$ within $\mathrm{t}=1 \mathrm{~s}$ $\sim \mathrm{t}=5 \mathrm{~s}$ (Fig. 6). In contrast to the $\mathrm{NaCl}$ solution, the $5 \%$, $\mathrm{CuSO}_{4} \cdot 5 \mathrm{H}_{2} \mathrm{O}$ solution with lower electrical conductivity (30 $\mathrm{ms} / \mathrm{cm}$ ) induced less working current which slowed the growth of the bubbles to $t=5 \mathrm{~s}$ (Fig. 5). Here, a steady current of $0.8 \mathrm{~A}$ (Fig. 6) and a final static head of $5 \mathrm{~mm}$ (Fig. 7) were observed.

Compared to Figs. 6 and 7, similar distributions are shown in maximum head and working current with time. For the $\mathrm{NaCl}$ solution, the head rapidly climbs to a peak value of $14 \mathrm{~mm}$ in the first second (Fig. 7). This rise is analogous to the current distribution in Fig. 6, where the maximum current approximately reaches $1.8 \mathrm{~A}$. Afterwards, the hydraulic head lowers to $2 \mathrm{~mm}$ instantaneously and then an unstable fluctuation between $2 \mathrm{~mm}$ 


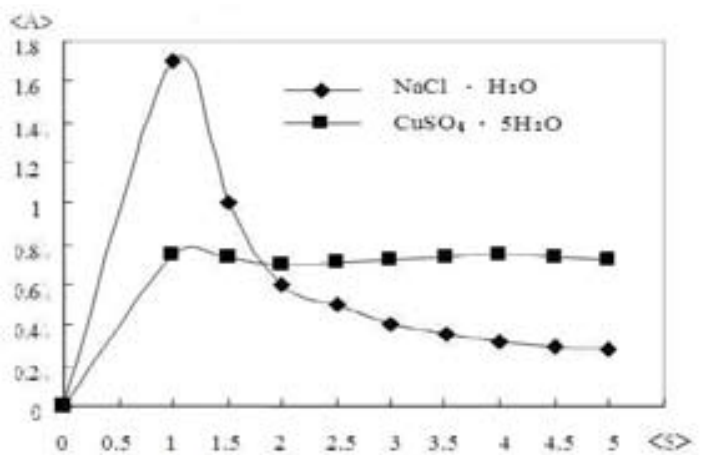

Fig. 6. Variation of induced current and time under an applied voltage of $9 \mathrm{~V}$.

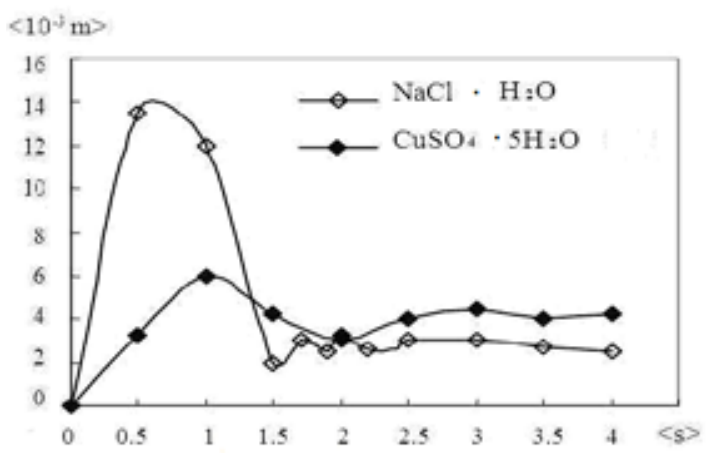

Fig. 7. Variation of hydraulic head and time under an applied voltage of $9 \mathrm{~V}$.

$\sim 3 \mathrm{~mm}$ occurred before the final static head reaches in the next 2 seconds (Fig. 7). This result corresponds to the decline of the induced current in Fig. 6 where the weaker Lorentz force is no longer able to support the liquid medium, so the hydraulic head drops down immediately with a sharp fluctuation (Fig. 7). As for the discussion in $\mathrm{CuSO}_{4} \cdot 5 \mathrm{H}_{2} \mathrm{O}$ solution, the hydraulic head in Fig. 7 gradually increases to a maximum value of $6 \mathrm{~mm}$ initially and shows a slow decrease with a slight fluctuation in five seconds. This result also agrees with the current distribution in Fig. 6, where the induced current slowly rises to $0.8 \mathrm{~A}$ and then remains constant.

\section{Maximum Head, Analytic Head, and Dynamic Head with the Induced Current}

In dealing with the maximum head and induced current in $\mathrm{NaCl}$ solution, Fig. 8 shows that the maximum head increases linearly as the current varies $(0.4 \mathrm{~A}, 4 \mathrm{~mm} \sim 1.8 \mathrm{~A}, 15 \mathrm{~mm})$. The analytic head with its final static state also presents a linear relationship with the induced current $(0.4 \mathrm{~A}, 2 \mathrm{~mm} \sim 1.8 \mathrm{~A}$, $9 \mathrm{~mm})$. The discrepancy between both distributions can be defined as the dynamic head caused by initial fluid acceleration with active Lorentz force. Therefore kinetic energy in the dynamic head can be determined by the working current and faster flow velocity. As for $\mathrm{CuSO}_{4} \cdot 5 \mathrm{H}_{2} \mathrm{O}$ solution in Fig. 9, both distributions of the maximum head $(0.2 \mathrm{~A}, 2 \mathrm{~mm} \sim 0.8 \mathrm{~A}, 6 \mathrm{~mm})$ and the analytic head $(0.2 \mathrm{~A}, 1.7 \mathrm{~mm} \sim 0.8 \mathrm{~A}, 4 \mathrm{~mm})$ are so close that the difference between them is insignificant, yet both will slightly increase with an induced current. This leads to the

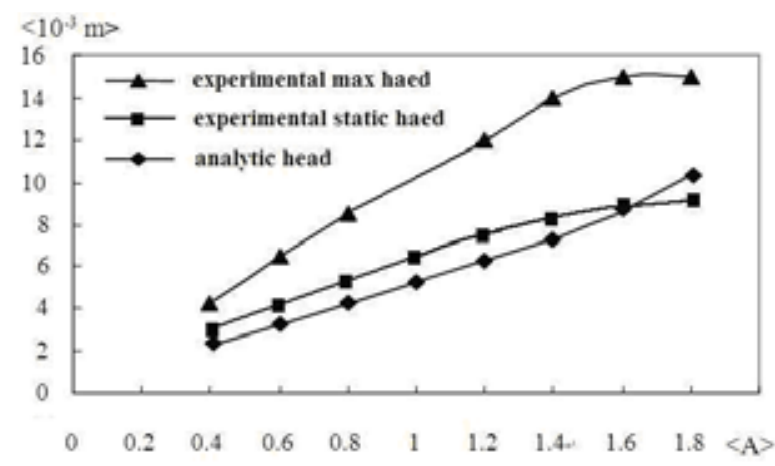

Fig. 8. Comparison between maximum head, static head, and analytic head with maximum current for $\mathrm{NaCl} \cdot \mathrm{H}_{2} \mathrm{O}$.

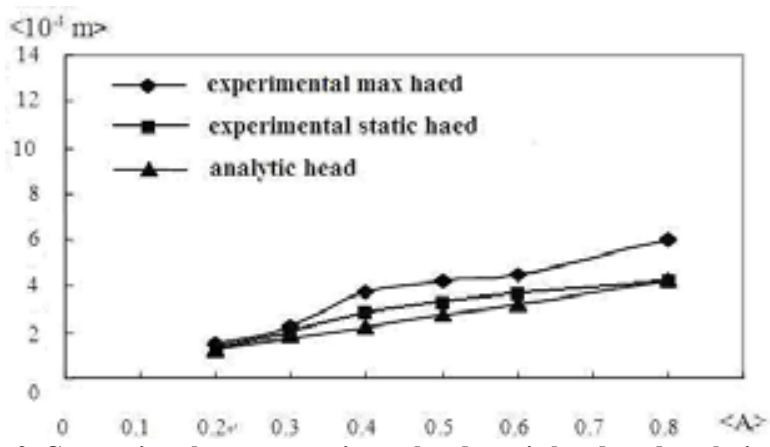

Fig. 9. Comparison between maximum head, static head, and analytic head, with maximum current for $\mathrm{CuSO}_{4} \cdot 5 \mathrm{H}_{2} \mathrm{O}$.

prediction that the dynamic head of the lower conducting medium, $\mathrm{CuSO}_{4} \cdot 5 \mathrm{H}_{2} \mathrm{O}$, is small and nearly independent of the induced current in the pumping process. Therefore the liquid will be slowly and steadily elevated, which is dissimilar to the result in higher electrically conductivity solution $(\mathrm{NaCl})$.

Figs. 8 and 9 also indicate that the analytic solutions in equation (2) are well in agreement with the experimental static heads. That implies an easier option by using the simpler equation to determine the static head and its availability in this study.

\section{Maximum Head with Aqueous Concentration in Dif- ferent Voltage Applied}

The maximum head distribution of $\mathrm{NaCl}$ solution with a concentration of $1 \%$ and $5 \%$ is discussed in Fig. 10. Though both distributions nearly keep a linear relation with the applied voltage, the maximum heads of $0 \mathrm{~mm} \sim 2 \mathrm{~mm}$ in a $1 \%$ concentration are much less than those of $0 \mathrm{~mm} \sim 16 \mathrm{~mm}$ in a $5 \%$ concentration and their difference extends as voltage increases $(0$ $\mathrm{mm}, 1 \mathrm{~V} \sim 13 \mathrm{~mm}, 9 \mathrm{~V})$. A denser solution with higher electrical conductivity produces stronger instantaneous Lorentz force to raise the working medium and makes a result of significant deviation for the maximum head in both cases; especially under the condition of higher applied voltage. In contrast to the above discussion, both maximum heads in $1 \%$ and $5 \% \mathrm{CuSO} 4 \cdot 5 \mathrm{H} 2 \mathrm{O}$ solutions slightly vary with the increase of applied voltage (Fig. 11). Their difference, $0 \mathrm{~mm} \sim 3 \mathrm{~mm}$, is relative small and nearly insensitive to variation of concentrations. This smaller divergence tells that the weak MHD effect will be not much improved 


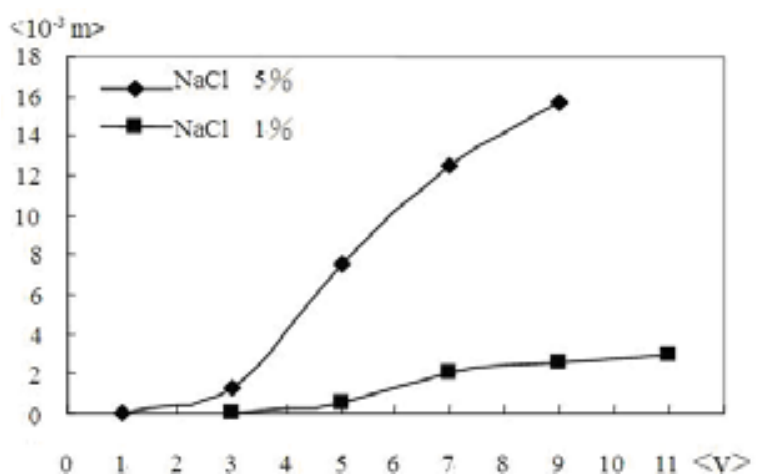

Fig. 10. Comparison between the maximum head of $5 \% \mathrm{NaCl}$ and $1 \% \mathrm{NaCl}$ solutions with an applied voltage of $3 \mathrm{~V}-11 \mathrm{~V}$.

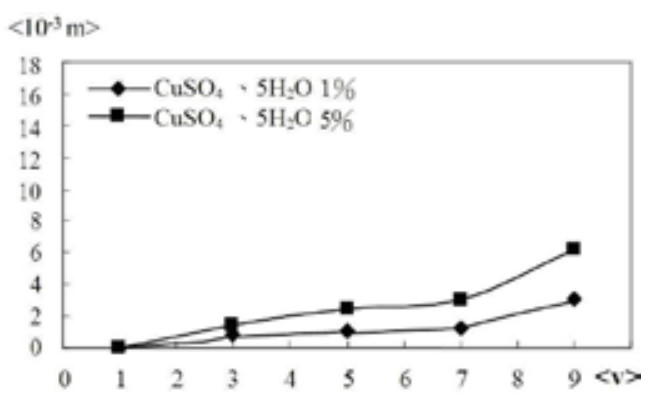

Fig. 11. Comparison between the maximum head of $1 \%$ and $5 \%$ $\mathrm{CuSO}_{4} \cdot 5 \mathrm{H}_{2} \mathrm{O}$ solutions with an applied voltage of $3 \mathrm{~V}-11 \mathrm{~V}$.

with lower electrical conductivity solution of $\mathrm{CuSO} 4 \cdot 5 \mathrm{H} 2 \mathrm{O}$, even in the usage of higher concentrations.

\section{CONCLUSION}

Several important results from above discussion are concluded as follows:

1. Bubbles produced in higher induced current not only retard flow motion, but also lower the hydraulic head.

2. Due to the required low flow rate in the minipump, lower input voltage with weaker magnetic intensity is adequate to produce Lorentz force to overcome the flow drag and reduce the birth of bubbles.

3. Maximum heads with a time in higher electrical conductivity solution are similar to the distribution of delta function and suitable to be applied in an impulse pump. Nevertheless, the stable growth of the maximum heads in a lower electrical conductivity solution fits the continuous type pump.

4. With the expansion of applied voltage, the height of both maximum and dynamic heads will be significantly enhanced in higher electrical conductivity solution.

5. A simplified steady solution in an analytic model is of special advantage to determine the static head which has been proven to be in excellent agreement with the experimental data.

\section{NOMENCLATURE}

$\sigma \quad$ : surface tension coefficient

$\rho \quad$ : density of working medium

$r \quad$ : radius of static tube

$h_{0}:$ surface tension head

$J \quad$ : induced current density

$B$ : magnetic flux density

$L \quad$ : the length of the test channel

$\Delta h:$ Lorentz static head

\section{REFERENCES}

1. Geng, X., Yuan, H., and Öguz, H. N., "Bubble-based micro pump for electrically conducting liquids," Journal of Micromechanics and Microengineering, Vol. 11, pp. 270-276 (2001).

2. Huang, L., Wang, W., Murphy, M. C., Lina, K., and Ling, Z. G.., "Fabrication and test of a de type magneto-hydrodynamic (MHD) micro-pump," Microsystem Technology, Vol. 6, pp. 235-240 (2000).

3. Jang, J. and Lee, S. S., "Theoretical and experimental study of MHD (magneto hydro dynamic) micro pump," Sensors and Actuators, ,Vol. 80, pp. 84-89 (2000).

4. Luis, S., Verardi, L., Cardoso, J. R., and Costa, M. C., "Three-dimensional finite element analysis of MHD duct flow by the penalty function formulation," IEEE Transactions on Magnetics, Vol. 37, pp. 384-390 (2001).

5. Wang, P. J., Chang, C. Y., and Chang, M. L., "Simulation of two-dimensional fully developed laminar flow for a magneto-hydrodynamic (MHD) pump," Biosensors and Bioelectronics, Vol. 20, pp. 115-121 (2004). 
